\title{
The polish wheat (Triticum polonicum L.) TpSnRK2.10 and TpSnRK2.11 meditate the accumulation and the distribution of $\mathrm{cd}$ and Fe in transgenic Arabidopsis plants
}

Ruijiao Wang ${ }^{1+}$, Chao Wang ${ }^{1+}$, Qin Yao ${ }^{1}$, Xue Xiao ${ }^{1}$, Xing Fan ${ }^{1,2}$, Lina Sha ${ }^{1,2}$, Jian Zeng ${ }^{3}$, Houyang Kang ${ }^{1,2}$, Haiqin Zhang ${ }^{1,2}$, Yonghong Zhou ${ }^{1,2}$ and Yi Wang ${ }^{1,2^{*}}$

\begin{abstract}
Background: The SnRK2s (Plant specific protein kinase) are involved in various biological processes, such as plant defense and environmental challenges. In Arabidopsis, AtSnRK2s regulate the expression of some metal transporters. For example, AtSnRK2.4 plays a role in the regulation of Arabidopsis tolerance to Cd; AtSnRK2.2 and AtSnRK2.3 are involved in Cd uptake and translocation. However, the functions of their homologs, TpSnRK2.10 and TpSnRK2.11 from dwarf Polish wheat are unknown.

Results: TpSnRK2.11 encodes a cytoplasmic protein. TpSnRK2.10 and TpSnRK2.11 have different expression patterns at different growth stages. Expression of TpSnRK2.10 increased yeast's sensitivity to $\mathrm{Cd}$; conversely, expression of TpSnRK2.11 enhanced yeast's tolerance to Cd. Overexpression of TpSnRK2.10 or TpSnRK2.11 did not affect Cd sensitivity in Arabidopsis, but significantly increased $\mathrm{Cd}$ accumulation in roots and shoots, and $\mathrm{Cd}$ translocation from roots to shoots. While, Fe accumulation was significantly increased in roots but decreased in shoots by overexpression of TpSnRK2.10; opposite results were observed in TpSnRK2.11-overexpressing lines. Subcellular distribution analysis found that overexpression of TpSnRK2.10 and TpSnRK2.11 increased Cd concentration in cell wall and organelle fractions of roots and shoots; meanwhile, they also differentially influenced Fe distribution.

Conclusions: These results indicated that TpSnRK2.10 and TpSnRK2.11 are involved in the uptakes and the translocations of $\mathrm{Cd}$ and Fe, possibly by regulating the expression of AtNRAMP1 and AtHMA4, and other genes involved in the synthesis of phytochelatins or hemicellolosic polysaccharides.
\end{abstract}

Keywords: Wheat, TpSnRK2, Cd, Biological function, Expression pattern

\section{Background}

More arable land soils have been contaminated by cadmium $(\mathrm{Cd})$ through various industrial processes and/or agricultural practices [1]. As one of the toxic heavy metals, $\mathrm{Cd}$ is readily taken up by plants, and then entries into food chain, which potentially affects human health

\footnotetext{
*Correspondence: wangyi@sicau.edu.cn

${ }^{\dagger}$ Ruijiao Wang and Chao Wang contributed equally to this work.

${ }^{1}$ Triticeae Research Institute, Sichuan Agricultural University, Wenjiang 611130, Sichuan, China

${ }^{2}$ Joint International Research Laboratory of Crop Resources and Genetic Improvement, Sichuan Agricultural University, Wenjiang 611130, Sichuan, China

Full list of author information is available at the end of the article
}

[2]. In plants, $\mathrm{Cd}$ is absorbed via basal roots, loaded in the xylem, then transported to shoots [3,4], which negatively affects mineral nutrition and homeostasis in tissues and root growth and development $[2,5-7]$. Characterization of numerous $\mathrm{Cd}$ transporters responsible for $\mathrm{Cd}$ accumulation and transport found that they are regulated by intricate stress signal transduction pathways $[2,5]$. Thus, our knowledge about $\mathrm{Cd}$ accumulation and transport remains limited; the investigation is still needed to understand the molecular mechanisms.

The Cd signal transduction pathway is mediated by plant protein kinases that are major signal transduction

(c) The Author(s). 2019 Open Access This article is distributed under the terms of the Creative Commons Attribution 4.0 International License (http://creativecommons.org/licenses/by/4.0/), which permits unrestricted use, distribution, and reproduction in any medium, provided you give appropriate credit to the original author(s) and the source, provide a link to the Creative Commons license, and indicate if changes were made. The Creative Commons Public Domain Dedication waiver (http://creativecommons.org/publicdomain/zero/1.0/) applies to the data made available in this article, unless otherwise stated. 
elements [8, 9]. For example, $\mathrm{Cd}$ stress induces the activities of mitogen-activated protein kinases and sucrose nonfermenting-1 (SNF1)-related protein kinase 2 (SnRK2) in plants $[6,8,9]$. As plant specific protein kinase, ten SnRK2s are individually identified from Arabidopsis. Molecular analysis of them indicated that they are involved in various biological processes, such as plant defense to biotic and/or abiotic challenges [6, 10-12]. AtSnRK2.2 and AtSnRK2.3 regulate abscisic acid (ABA) response element (ABRE)-driven gene expression through the phosphorylation of ABRE binding factors (ABFs) [10]. Knockout of AtSnRK2.2 and AtSnRK2.3 enhances the expression of IRON-REGULATED TRANSPORTER1 (IRT1) under $10 \mu \mathrm{M}$ Cd stress [12]. IRT1 is a key transporter responsible for Cd uptake $[3,13]$. The knockout of IRT1 reduced $\mathrm{Cd}$ concentration in roots and increases in shoots [12], which is not consistent with its enhanced expression. Thus, knockout of AtSnRK2.2 and AtSnRK2.3 may regulate other metal transporters responsible for $\mathrm{Cd}$ uptake and translocation, such as AtNRAMPs whose promoters have an ABF or ABFs in Arabidopsis [14, 15]. In contrast, although knockout of AtSnRK2.4 did not affect the expression of IRT1 and the concentration of $\mathrm{Cd}$ under $20 \mu \mathrm{M}$ Cd stress, it enhanced Cd tolerance and reduced phytochelatins concentration [6]. Phytochelatins chelate $\mathrm{Cd}$ to form phytochelatin-Cd complexes, which are transported into vacuoles to enhance Cd tolerance [16]. Meanwhile, AtSnRK2.6 probably participates in multiple signaling pathways. Inactivation of AtSnRK2.6 impairs stomatal close to enhance transpiration [17]. Overexpression of AtSnRK2.6 increases the content of fructose and glucose [18]. Hemicellolosic polysaccharides of cell wall, consisted of fructose, glucose and other sugars, are major Cd binding sites, and positively correlate with the acquire capacity of Cd [19]. These results indicate that SnRK2s could be involved in Cd uptake and translocation; meanwhile, their mechanisms and functions may be different.

Several SnRK2s from rice and wheat, including OsSAPK8, OsSAPK9, OsSAPK10 and TaPKABA1, can also phosphorylate ABFs [20, 21]. Thus, they also potentially regulate the expression of genes with an ABRE or ABREs in their promoters. In wheat, 10 SnRK2s (TaSnRK2.1-TaSnRK2.10) are isolated and grouped into three subclasses. Subclass III TaSnRK2s are regulated by ABA stress, suggesting that these genes are involved in ABA regulated stress responses; however, subclass I and II TaSnRK2s are regulated by PEG, $\mathrm{NaCl}$ and cold stress, but not by ABA stress, suggesting that these genes responded to various abiotic stressors in an ABA-independent manner [22]. In tetraploid wheat, eight $S n R K 2 s$ (TpSnRK2.1, 2.2, 2.3, $2.5,2.7,2.8,2.10$ and 2.11 ) are cloned from Polish wheat (Triticum polonicum L.); except of TpSnRK2.7, all of the genes are differentially regulated by ABA,
$\mathrm{NaCl}, \mathrm{PEG}$ and cold stress [23, 24]. However, whether these genes involved in $\mathrm{Cd}$ uptake and translocation are still unknown. Since TpSnRK2.10 is homologous with $A t S n R K 2.2$ and $A t S n R K 2.3$, and $T p S n R K 2.11$ is homologous with $A t S n R K 2.4$, we hypothesized that they are involved in $\mathrm{Cd}$ uptake, translocation and tolerance. To test these hypotheses, we investigated their biological functions by analyzing expression pattern, subcellular localization in Arabidopsis leaf protoplast, $\mathrm{Cd}$ tolerance in yeast, and the concentration and subcellular distribution of $\mathrm{Cd}, \mathrm{Fe}$ and $\mathrm{Zn}$ in Arabidopsis overexpressing lines.

\section{Methods \\ Materials}

Dwarf Polish wheat (Triticum polonicum L., $2 \mathrm{n}=4 \mathrm{X}=28$, AABB, DPW) used in the present study was collected from Tulufan of Xinjiang province, China. Previous study indicated that the seedling of DPW exhibits high tolerance to $\mathrm{Cd}$ [25]. Thus, it is a desirable material for analyzing the mechanism of high tolerance to Cd. The seed of Arabidopsis thaliana (wild type, WT) was provided by Professor Yan Huang (College of Life Science, Sichuan Agricultural University).

\section{Bioinformatics and phylogenetic analysis}

The full length cDNAs of TpSnRK2.10 (KF688099) and TpSnRK2.11 (KF688100) amplified from DPW were described by Wang et al. [23]. Their gene structures and chromosome localizations were analyzed using BLAST to search against the genome of Triticum aestivum [26] on website of Ensemblplants (http://plants.ensembl.org/Triticum_aestivum/Tools/Blast?db=core). Their putative subcellular localizations were predicted by the ProtComp 9.0 (http://linux1.softberry.com/berry.phtml?group=programs\&subgroup $=$ proloc\&topic $=$ protcomppl). The software of Vector NTI 11.5.1 (Invitrogen, Carlsbad, CA, USA) was used to align amino acid sequences including TpSnRK2.10, TpSnRK2.11, AtSnRK2.2, AtSnRK2.3 and AtSnRK2.4.

\section{Expression analysis of TpSnRK2.10 and TpSnRK2.11}

For expression pattern in DPW grown in normal growth season, tissues were collected from three growth stages with three biological replicates, including jointing stage (root, basal stem, leaf sheath, new leaf and young leaf), booting stage (root, basal stem, second stem, first node, second node, third node, lower leaf, flag leaf sheath, flag leaf blade and peduncle), and grain filling stages (root, stem, first node, leaf sheath, flag leaf blade, lemma and grain). All tissues were snap frozen in liquid nitrogen and stored at $-80^{\circ} \mathrm{C}$ for RNA extraction.

For response to $\mathrm{Cd}$ stress and Fe-deficient in DPW seedlings, sterilized DPW seeds were germinated in dark at room temperature for four days. Uniform seedlings 
were cultured in Hoagland's nutrient solution in a growth chamber for two weeks. All seedlings were treated with two stresses including $80 \mu \mathrm{M} \mathrm{CdCl}$ (Cd), and Fe-free Hoagland's nutrient solution (Fe-deficient). Seedlings grown in Normal Hoagland's nutrient solution were used as control (CK). After a week of treatments, roots were collected with three biological replicates and snap frozen in liquid nitrogen for RNA extraction.

Total RNA of each sample was isolated using the Total RNA Kit II (Omega, USA) according to the user manual. RNA concentration was measured by NanoDrop ND-1000 spectrophotometer (NanoDrop Technologies). The first-strand cDNA was synthesized from $2 \mu \mathrm{g}$ total RNA using the M-MLV First Strand cDNA Synthesis Kit (Omega, USA).

Relative expression of TpSnRK2.10 and TpSnRK2.11 was normalized as described by Wang et al. [23] that included the information of TpSnRK2.10- and TpSnRK2.11-specific primers, and reference gene (actin).

\section{Subcellular localization of TpSnRK2.11 in Arabidopsis protoplasts}

Subcellular localization of TpSnRK2.11 was analyzed as described by Wang et al. [4] and Peng et al. [27]. Briefly, the open reading frame of $T p S n R K 2.11$ was sub-cloned into the KpnI and XbaI sites of the Arabidopsis protoplast expression vector HBT95-GFP using TpSnRK2.11 primers (F:CGGGGTACCATGGACAAGT ACGA GGAGG, and R: GCTCTAGATTAGATGTGCAACGCG CTCA). Arabidopsis leaf mesophyll protoplast was prepared and transformed according to the method of Yoo et al. [28]. Signal of GFP was detected by a confocal laser scanning microscope (Olympus, Tokyo, Japan).

\section{Cd sensitive analysis in yeast}

$\mathrm{Cd}$ sensitivity in yeast was tested as described by Peng et al. [27]. Briefly, the open reading frames of TpSnRK2.10 and $T p S n R K 2.11$ were individually sub-cloned into the $K p n I$ and $X b a I$ sites of yeast expression vector pYES2 using $T p S n R K 2.11$ primers and $T p S n R K 2.10$ primers (F: CGGGGTACCATGGACCGGGCGGCGC, and R: TGCT C TAGATCACATAGCATACAC). The S. C. EasyComp Transformation kit (Invitrogen, USA) was used to transform plasmid into yeast strains including wild type (BY4743) and Cd sensitive strain (ydr135c) according to its user manual.

For $\mathrm{Cd}$ sensitive testing on synthetic defined (SD) plate medium, positive cell suspension with optical density $\mathrm{OD}_{600}=0.6$ was diluted to four sequential dilutions (1:10, 1:100, 1:1000, and 1:10000). Diluted cell suspensions $(5 \mu \mathrm{L})$ were cultured on SD plate medium containing $2 \%$ glucose or galactose, and $\mathrm{CdCl}_{2}(0 \mu \mathrm{M}, 80 \mu \mathrm{M}$, and $100 \mu \mathrm{M})$, at $30^{\circ} \mathrm{C}$ for 4 days.
For Cd sensitive testing in SD liquid medium, diluted cell suspensions $(5 \mu \mathrm{L}, 1: 10000)$ were cultured on SD liquid medium containing $2 \%$ galactose and $\mathrm{CdCl}_{2}(0 \mu \mathrm{M}$ and $80 \mu \mathrm{M})$, at $30^{\circ} \mathrm{C}$ with shaking at $250 \mathrm{rpm}$. $\mathrm{OD}_{600}$ values were investigated at $0,2,4,6,8,16,24,32,40$ and $48 \mathrm{~h}$ using a NanoDrop ND-1000 spectrophotometer (NanoDrop Technologies). All values were measured with three biological replicates.

\section{Functional analysis of TpSnRK2.10 and TpSnRK2.11 in overexpressing Arabidopsis lines}

Biological functions of TpSnRK2.10 and TpSnRK2.11 were analyzed in Arabidopsis overexpressing lines as described by Peng et al. [27]. Briefly, the open reading frames of TpSnRK2.10 and TpSnRK2.11 were sub-cloned into the $K p n I$ and $X b a I$ sites of the expression vector pCAMBIA1305.1 driven by the $35 \mathrm{~S}$ promoter. $T p S n R K 2.10$ and TpSnRK2.11 were individually transformed into WT Arabidopsis thaliana plants using floral infiltration via Agrobacterium-mediated transformation [29] (two time-independent transformations). Homozygous overexpressing lines were selected using hygromycin selection and PCR with gene-specific primers [23]. The relative expression of TpSnRK2.10 and TpSnRK2.11 in their individually transgenic lines was analyzed as described as the section of "Expression analysis of TpSnRK2.10 and TpSnRK2.11". Meanwhile, the relative expression of AtIRT1, AtNRAMP1 and AtHMA4 was also investigated in WT, vector line, TpSnRK2.10- and TpSnRK2.11-expressing lines using AtActin2 as reference gene. The qPCR primers of AtIRT1, AtNRAMP1, AtHMA4 and AtActin 2 were used as described by Ihnatowicz et al. [30], Boonyaves et al. [31] and Chen et al. [32], respectively.

For investigation of $\mathrm{Cd}$ sensitive in TpSnRK2.10- and $T p S n R K 1.11$-expressing lines, sterilized seeds of WT, an empty vector line and independent homozygous lines (each line contained three plant lines selected from an independent transformation; totally six plant lines) were germinated on $1 / 2 \mathrm{MS}$ solid plates containing $\mathrm{CdCl}_{2}$ $(0 \mu \mathrm{M}$ and $40 \mu \mathrm{M})$ in a light incubator with $120 \mu \mathrm{E} \mathrm{m} \mathrm{m}^{-2}$ $\mathrm{s}^{-1}$ illumination intensity, $16 / 8 \mathrm{~h}$ light/dark period, $22^{\circ} \mathrm{C}$ temperature and $50 \%$ humidity. On the tenth day, root length of each line was measured.

To investigate whether TpSnRK2.10 and /or TpSnRK2.11 influenced metal $(\mathrm{Cd}, \mathrm{Fe}$ and $\mathrm{Zn})$ concentration in TpSnRK2.10- and TpSnRK1.11-overexpressing lines, uniform seedlings of WT, an empty vector line and independent homozygous lines were cultured in soil. After three weeks, soil was added once with $\mathrm{CdCl}_{2}(0 \mathrm{mg} / \mathrm{kg}$ and 40 $\mathrm{mg} / \mathrm{kg}$ ) dissolved in water. After four weeks, dry weight of each plant was measured. Part of roots and shoots was dried at $80^{\circ} \mathrm{C}$ for two days to measure metal concentration as described by Cheng et al. [7]. Remaining roots and 
shoots were used to measure the subcellular distribution of $\mathrm{Cd}$ and Fe as described by Cheng et al. [7].

\section{Data analysis}

All data was statistically analyzed using Tukey's test at $P \leq 0.05$ in SPSS 20.0. Figures were drawn by the software of SigmaPlot 12.0.

\section{Results}

\section{Chromosome localizations and gene structures of} TpSnRK2.10 and TpSnRK2.11

Blast search of the open reading frames of TpSnRK2.10 and TpSnRK2.11 against the genome of $T$. aestivum revealed that TpSnRK2.10 is located on chromosome 4AL (gene: TRIAE_CS42_4AL_TGACv1_291776_AA0996560) and comprises eight exons and seven introns; TpSnRK2.11 is located on chromosome 1BL (gene: TRIAE_CS42_1BL_TGACv1_031102 AA0107780) and comprises nine exons and eight introns.

\section{Homology analysis of TpSnRK2.10 and TpSnRK2.11 with AtSnRK2.2, AtSnRK2.3 and AtSnRK2.4}

Previously phylogenetic analysis revealed that TpSnRK2.10 grouped with AtSnRK2.2 and AtSnRK2.3, which belong to the subgroup 3 kinases that are strongly activated by ABA; TpSnRK2.11 grouped with AtSnRK2.4, which belongs to the subgroup 1 kinases that are not activated by ABA.
Alignment found that TpSnRK2.11 had a $68.6 \%$ identity to AtSnRK2.4 (Fig. 1a); TpSnRK2.10 had a 76.7 and $80.7 \%$ identity to AtSnRK2.2 and AtSnRK2.3, respectively (Fig. 1b). Further analysis revealed that except for $\mathrm{N}$-myristoylation site, other protein kinases motifs (including ATP-binding region, serine/threonine protein kinases activity site, activation loop, and transmembrane helix site) had one and/or more than one residue differences between TpSnRK2.11 and AtSnRK2.4 (Fig. 1a). While, only three protein kinases motifs (including ATP-binding region, transmembrane helix site, and required for $\mathrm{ABA}$ responses site) had residue differences among TpSnRK2.10, AtSnRK2.2 and AtSnRK2.3 (Fig. 1b). These residue differences in motifs implied functional divergence between TpSnRK2.11 and AtSnRK2.4, and among TpSnRK2.10, AtSnRK2.2 and AtSnRK2.3.

\section{Expression patterns of TpSnRK2.10 and TpSnRK2.11}

Expression patterns of TpSnRK2.10 and TpSnRK2.11 were investigated in different tissues at jointing, booting and grain-filling stages of wheat grown in a field. At jointing stage, expression of $T p S n R K 2.10$ was the highest in new leaves and young leaves, followed by leaf sheathes, and the lowest in basal stems (Fig. 2a). At booting stage, TpSnRK2.10 was expressed the highest in lower leaves, flag leaf sheathes and flag leaf blades, and the lowest in basal stems and second stems (Fig. 2a). At grain-filling stage, the highest expression of TpSnRK2.10

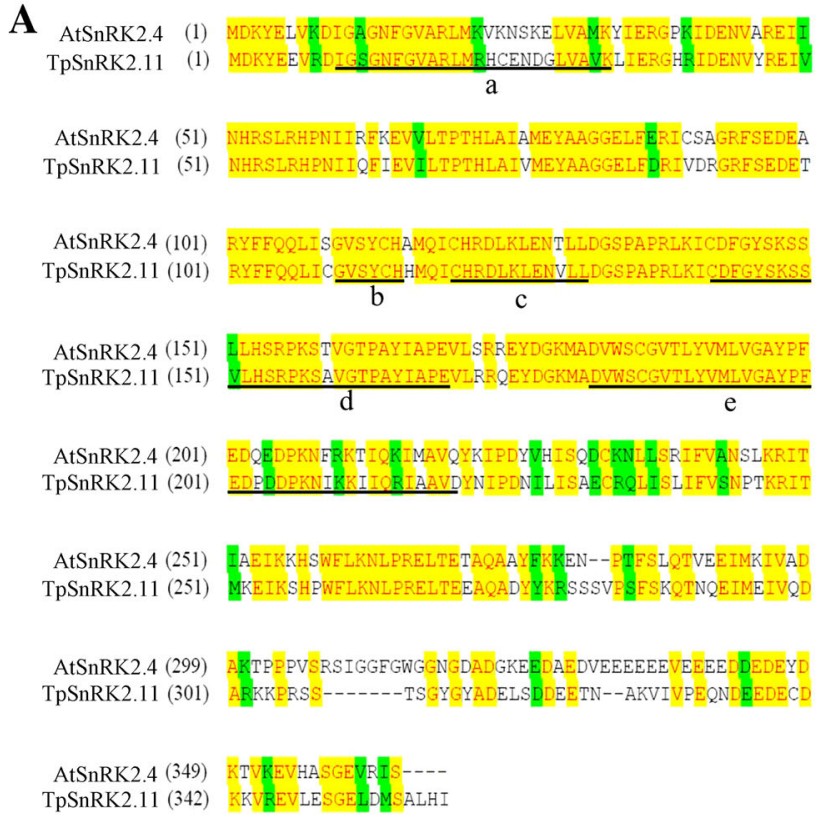

A AtSnRK2.4 (1) MDKYELVKDIGAG GNGGVARLMKVKNSKELVAMKYIERGPKIDENVAREII TpSnRK2.11 (1) MDKYEEVRD G GGGNFGVARTMRHCENDGIVAULLIERGHRIDENVYREIV a

AtSnRK2.4 (51) NHRSLRHENIIREKEVVLTPTHLAIAMEYAAGGELFERICSAGRESEDEA TpSnRK2.11 (51) NHRSLRHPNI IQFIEVILTPTHLAIVMEYAAGGELEDRIVDRGRESEDET

AtSnRK2.4 (101) RYEFQQLISGVSYCHAMQICHRDLKLENTLIDGSPAPRLKICDEGYSKSS TpSnRK2.11 (101) RYFEQQIICGVSYCHHMQICHRDLKLENVLIDGSPAPRLKITDFGYSKSS AtSnRK2.4 (151) LLHSRPKSTVGT PAYIAPEVISRREYDGKMADVWSCGVTLYYMLVGAYPE TpSnRK2.11 (151) d e

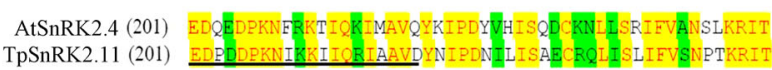
AtSnRK2.4 (251) TAEIKKHSWELKNLPRELTETAQAAYFKKEN--PMESLQTVEEIMKIVAD TpSnRK2.11 (251) MKEIKSHPWELKNLPRELTEEAQADYYKRSSSVVSESKQTNQEIMEIVQD

AtSnRK2.4 (299) ARTPEPVSRSIGGFGWGGNGDADGKEEDAEDVEEEEEEVEEEEDEEDEYL TpSnRK2.11 (301) ARKKRRSS------TSGYGYADELSDDEETN--AKVIVPEQNDEEDECD

AtSnRK2.4 (349) KTVREVHASGEVRTS----

TpSnRK2.11 (342) KKVREVLESGELDMSALHI

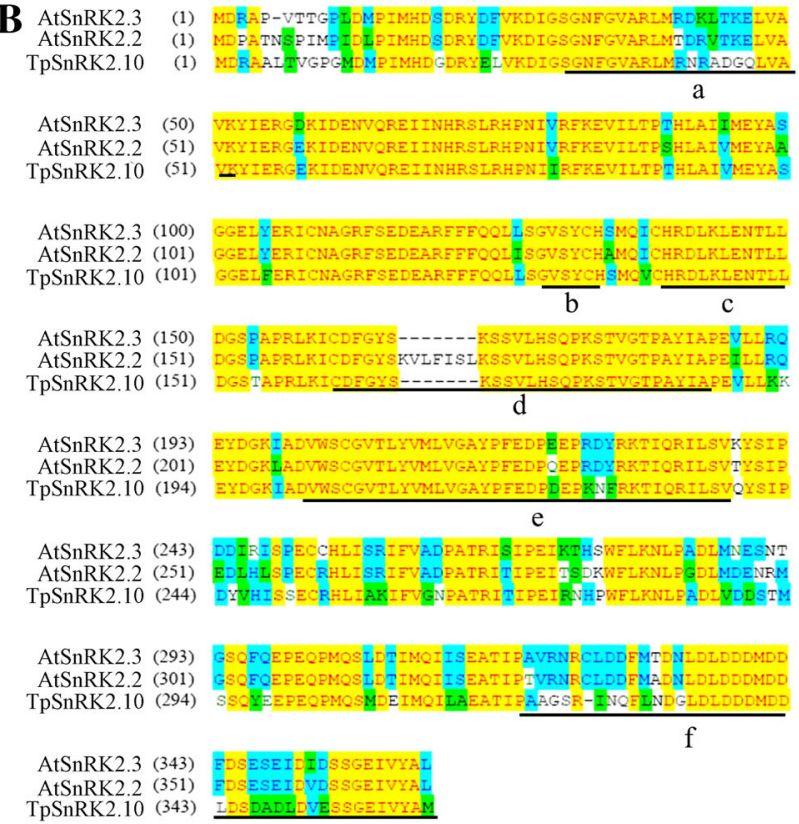

Fig. 1 Alignments of TpSnRK2.10, AtSnRK2.2 and AtSnRK2.3, and TpSnRK2.11 and AtSnRK2.4. a: alignment of the amino acid sequences of TpSnRK2.11 and AtSnRK2.4; b: alignment of the amino acid sequences of TpSnRK2.10, AtSnRK2.2 and AtSnRK2.3. a: ATP binding region; b: Nmyristoylation site; c: serine/threonine protein kinase activity site; d: activation loop; e: transmembrane helix site; f: required for ABA response 


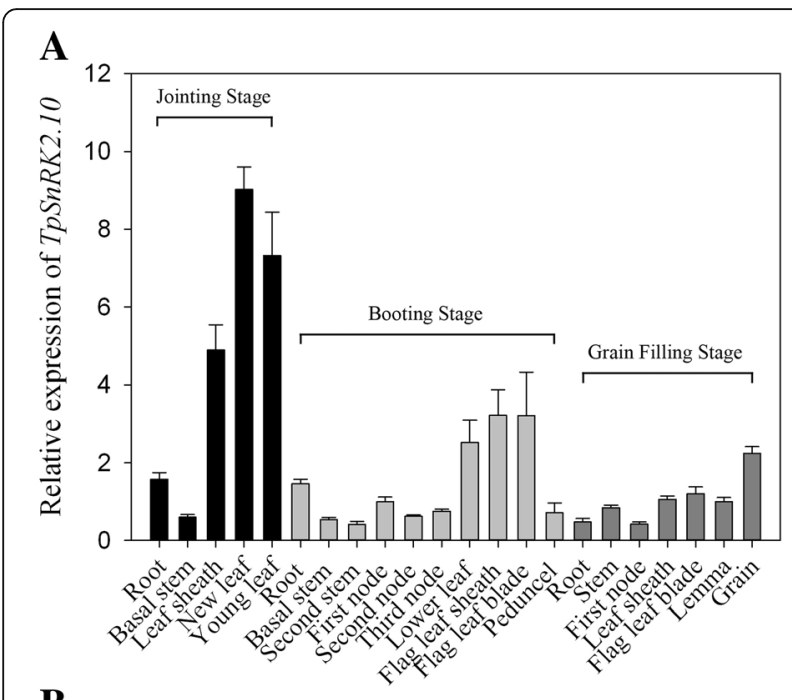

B

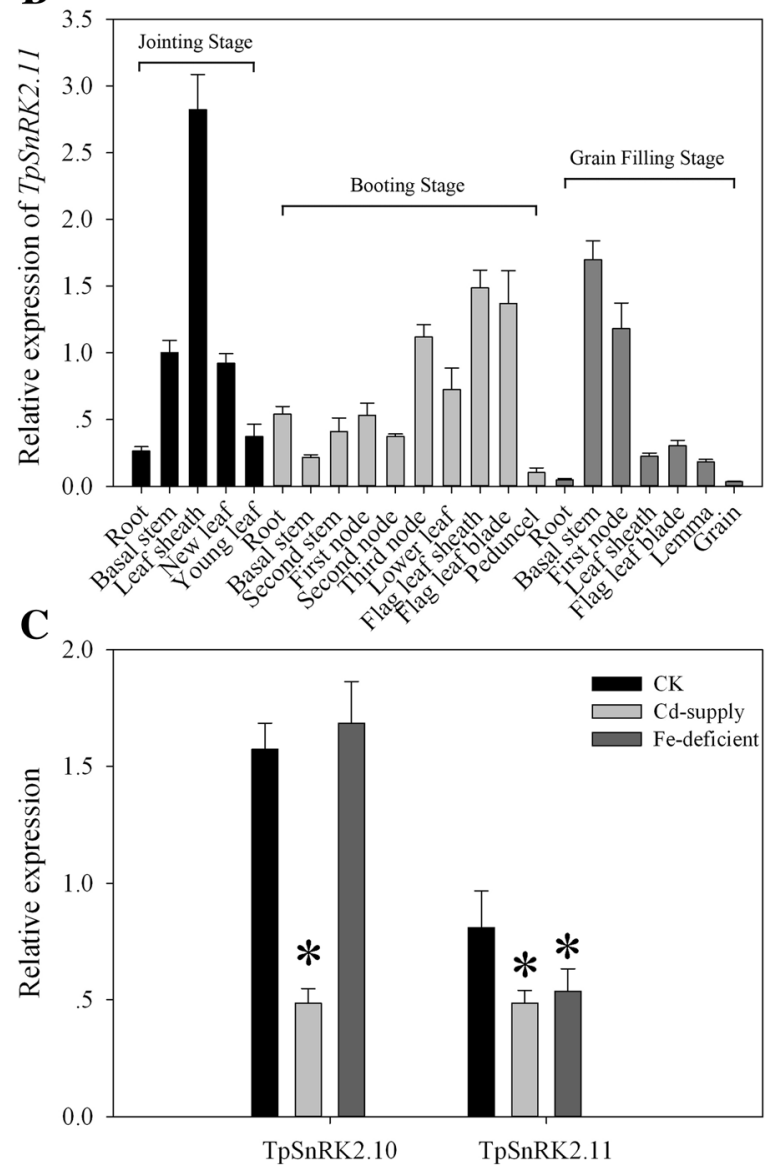

Fig. 2 Expression patterns of TpSnRK2.10 and TpSnRK2.11. a Expression level of TpSnRK2.10 in different tissues at three growth stages; $\mathbf{b}$ expression level of TpSnRK2.11 in different tissues at three growth stages; $\mathbf{c}$ expression levels of TpSnRK2.10 and TpSnRK2.11 under Cd-supply and Fe-deficient stresses. Jointing stage: the basal stem emerges above the soil line; booting stage: the developing head with the sheath of the flag leaf becomes visibly enlarged. Asterisk indicated significant difference when compared with WT at $P<0.05$; values were means \pm standard deviation (three biological replicates) was observed in grains, and the lowest was found in roots and first nodes (Fig. 2a).

The expression pattern of TpSnRK2.11 was different from that of TpSnRK2.10 (Fig. 2b). At jointing stage, the highest expression of TpSnRK2.11 was found in leaf sheathes, followed by in basal stems and new leaves, and the lowest was in roots and young leaves (Fig. 2b). At booting stage, $T p S n R K 2.11$ was mainly expressed in flag leaf sheathes, flag leaf blades and third nodes, then in lower leaves, roots and first nodes, and the lowest in basal stems and peduncels (Fig. 2b). At grain-filling stage, expression of $T p S n R K 2.11$ was the highest in basal stems and first nodes, and the lowest in roots and grains (Fig. 2b).

Meanwhile, the responses to Cd-supply and Fe-deficient were investigated (Fig. 2c). The expressions of TpSnRK2.10 and $T p S n R K 2.11$ were dramatically down-regulated by $\mathrm{Cd}$ stress when compared with their individually CK (Fig. 2c). The expression of TpSnRK2.10 was not regulated by Fe-deficient; while the expression of TpSnRK2.11 was down-regulated by Fe-deficient (Fig. 2c). These results indicate that TpSnRK2.10 and TpSnRK2.11 are response to $\mathrm{Cd}$ and $\mathrm{Fe}$.

\section{Subcellular localization of TpSnRK2.11}

TpSnRK2.10 and TpSnRK2.11 were predicated to localize to the cytoplasm and the nucleus. To confirm the predicated subcellular localizations, a HBT95-TpSnRK2.11-GFP fusion vector was transiently transformed into Arabidopsis leaf protoplasts. Our previous study indicated that green fluorescence of the empty vector HBT95-GFP is located to the cytoplasm, the nucleus and the plasma membrane [4]. In this study, the green fluorescence of HBT95-TpSnRK2.11-GFP in Arabidopsis leaf protoplasts was detected in the cytoplasm, but was not in the nucleus (Fig. 3). Thus, TpSnRK2.11 encodes a cytoplasmic protein. Unfortunately, we failed to detect the subcellular localization of TpSnRK2.10.

\section{Cd sensitivity in yeast}

To investigate whether TpSnRK2.10 and TpSnRK2.11 alter $\mathrm{Cd}$ sensitivity in yeast, we expressed TpSnRK2.10, TpSnRK2.11 or pYES2 in BY4743 and ydr135c. In the presence of $2 \%$ glucose that represses gene expression with the GAL1 promoter in pYES2 vector [33, 34], Cd stress significantly inhibited the growth of $y d r 135 \mathrm{c}$ when compared with BY4743 (Fig. 4a); meanwhile, similar growths were detected among ydr135c individually transformed with pYES2, TpSnRK2.10 and TpSnRK2.11 (Fig. 4a). In the presence of $2 \%$ galactose that induces gene expression with the GAL1 promoter in PYES2 vector, expression of $T p S n R K 2.11$ dramatically increased the growth of ydr135c when compared with ydr135c transformed with pYES2 under both of $80 \mu \mathrm{M}$ and $100 \mu \mathrm{M} \mathrm{CdCl}{ }_{2}$ stresses (Fig. 4b); while expression of 


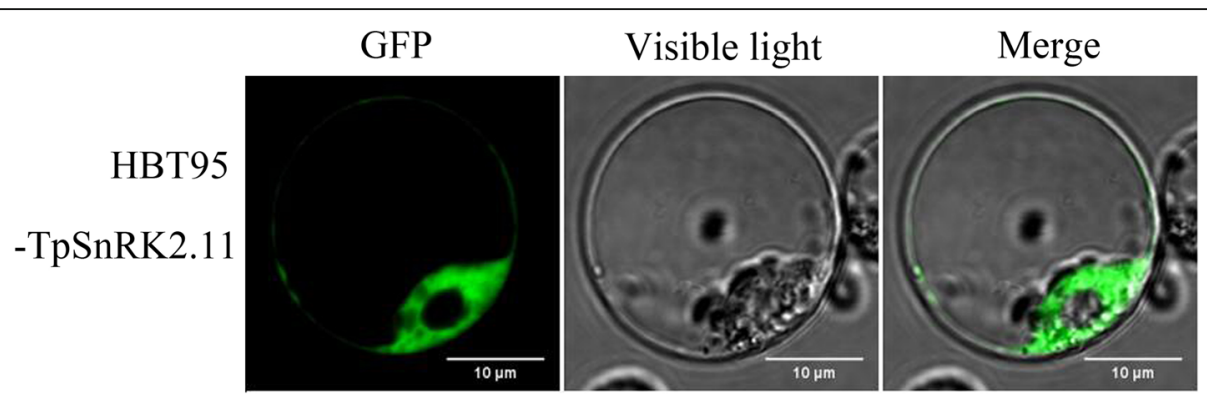

Fig. 3 Subcellular localization of TpSnRK2.11

A

pYES2-BY4743
pYES2-ydr135c
pYES2-TpSnRK2.10-BY4743
pYES2-TpSnRK2.11-BY4743
pYES2-TpSnRK2.10-ydr135c
pYES2-TpSnRK2.11-ydr135c

B

pYES2-BY4743
pYES2-ydr135c
pYES2-TpSnRK2.10-BY4743
pYES2-TpSnRK2.11-BY4743
pYES2- $T p S n R K 2.10$-ydr135c
pYES2- $T p S n R K 2.11$-ydr135c

C.

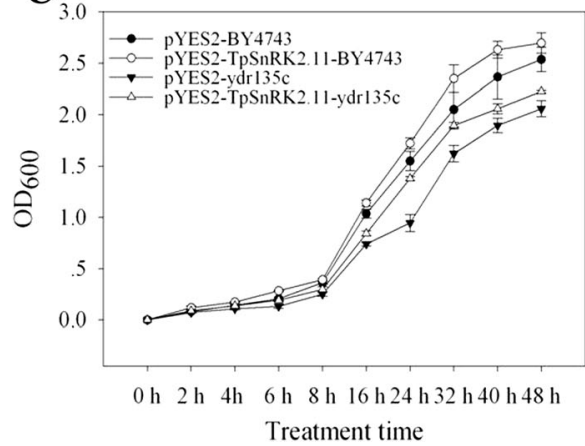

$\mathrm{CdCl}_{2}(\mu \mathrm{M}), 2 \%$ glucose

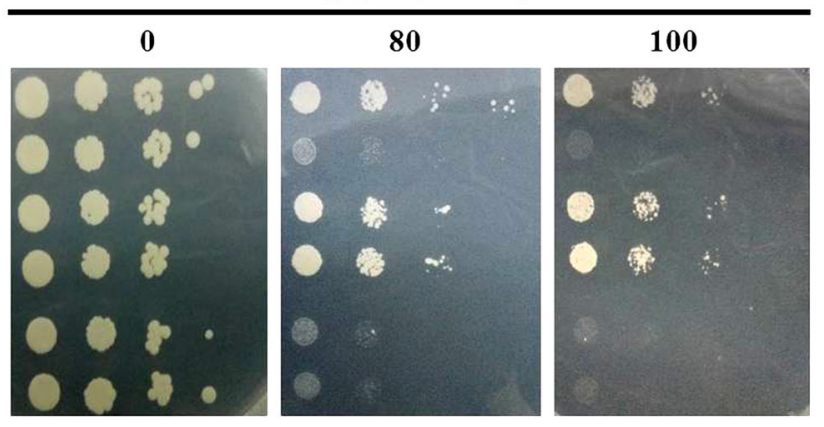

$\mathrm{CdCl}_{2}(\mu \mathrm{M}), 2 \%$ galactose

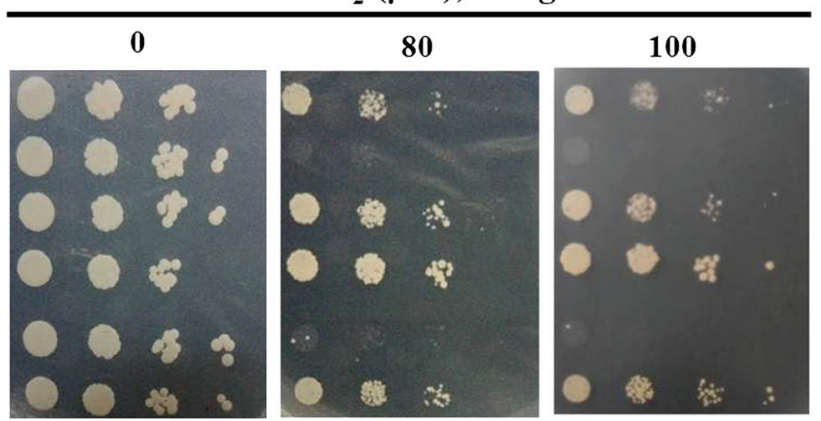

D

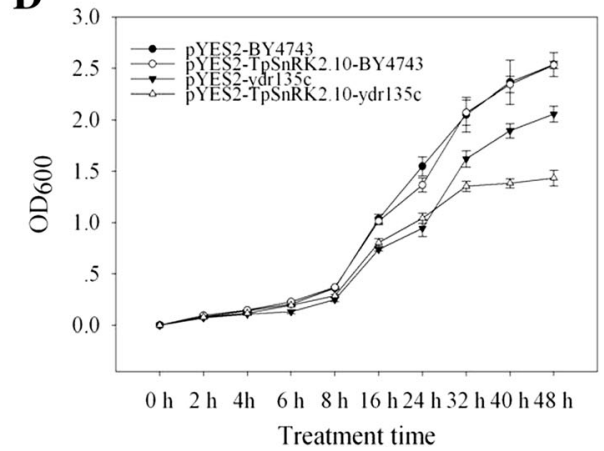

Fig. 4 Sensitivity to $C d$ in yeast. a-b Sensitivity to $C d$ when grown on plate SD medium with 0,80 or $100 \mu \mathrm{M} \mathrm{CdCl} 2$ under $2 \%$ glucose (A) or $2 \%$ galactose (B), at $30^{\circ} \mathrm{C}$ for 4 days; serial 1:10 dilutions were shown from left to right for yeast wild type (BY4743) and Cd sensitive strain (ydr135c) transformed with empty vector (pYES2), TpSnRK2.10 or TpSnRK2.11; c-d sensitivity to Cd when grown in liquid SD medium with $80 \mu \mathrm{M}$ CdCl 2 under $2 \%$ galactose; diluted cell suspensions ( $5 \mu \mathrm{L}, 1: 10000)$ were cultured from 0 to $48 \mathrm{~h}$ 
TpSnRK2.10 did not change the growth of ydr135c when compared with ydr135c transformed with pYES2 under $\mathrm{CdCl}_{2}$ stresses (Fig. 4b).

To confirm the results produced from plate testing, the growth curves of transformed yeast grown in liquid medium with $80 \mu \mathrm{M} \mathrm{CdCl} 2$ were investigated (Fig. 4c and d). Compared with ydr135c transformed with pYES2, expression of TpSnRK2.11 significantly increased the $\mathrm{OD}_{600}$ values of ydr135c starting at $16 \mathrm{~h}$ (Fig. 4c); while, expression of TpSnRK2.10 dramatically reduced the $\mathrm{OD}_{600}$ values of ydr135c staring at $32 \mathrm{~h}$ (Fig. $4 \mathrm{~d}$ ). These results indicated that expression of TpSnRK2.11 enhances $\mathrm{Cd}$ tolerance in yeast; while, expression of $T p S n R K 2.10$ increases Cd sensitivity in yeast.

\section{Functional overexpression of TpSnRK2.10 and TpSnRK2.11} in Arabidopsis

Since expression of TpSnRK2.10 and TpSnRK2.11 altered $\mathrm{Cd}$ sensitivity in yeast, it is interesting to investigate whether they would play roles in metal transport in plant. Thus, we individually overexpressed TpSnRK2.10 and
TpSnRK2.11 in Arabidopsis. Two independent homozygous of TpSnRK2.10- or TpSnRK2.11-overexpressing lines were developed (Additional file 1: Figure S1). When grown on 1/ 2 MS medium with $40 \mu \mathrm{M} \mathrm{CdCl}$, overexpression of TpSnRK2.10 or TpSnRK2.11 did not change root length compared with WT (Fig. 5a and b); Cd stress significantly inhibited root length when compared with CK (Fig. 5b). When grown in soil with $40 \mathrm{mg} / \mathrm{kg} \mathrm{CdCl}$, overexpression of TpSnRK2.10 or TpSnRK2.11 also did not change the dried weight of roots and shoots compared with CK (Fig. $5 \mathrm{c}$ and $\mathrm{d}$ ). These results indicated that overexpression of TpSnRK2.10 or TpSnRK2.11 did not influence Cd sensitivity in plant.

Meanwhile, we analyzed Cd, Fe and $\mathrm{Zn}$ concentrations in roots and shoots grown in soil. Under $40 \mathrm{mg} / \mathrm{kg}$ $\mathrm{CdCl}_{2}$ stress, overexpression of $T p S n R K 2.10$ or $T p S n R K 2.11$ significantly increased $\mathrm{Cd}$ concentration in roots and shoots when compared with WT and vector line (Fig. 6a and b). Under none metal stress, overexpression of TpSnRK2.10 significantly enhanced Fe concentration in roots (Fig. 6c), but reduced in shoots when

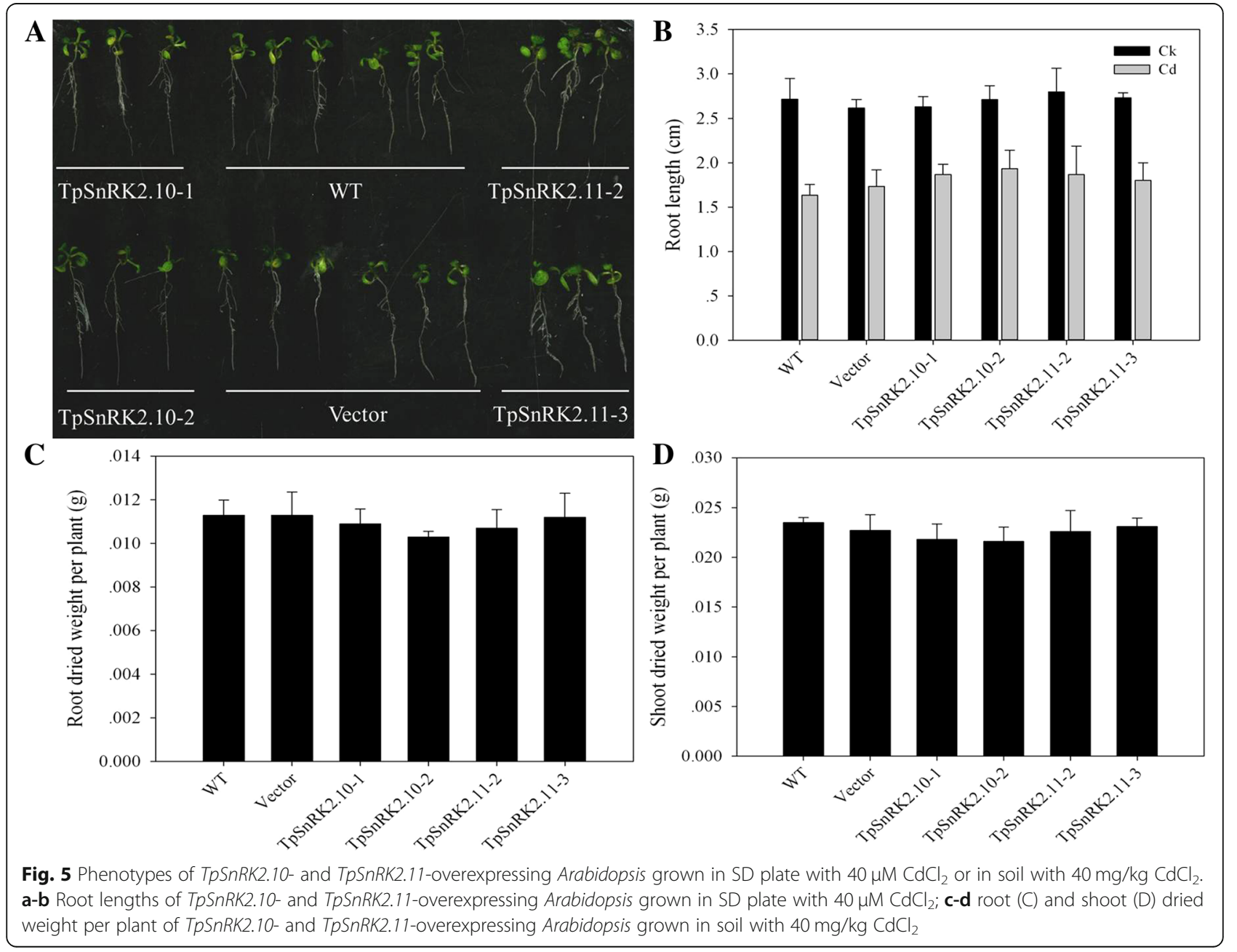



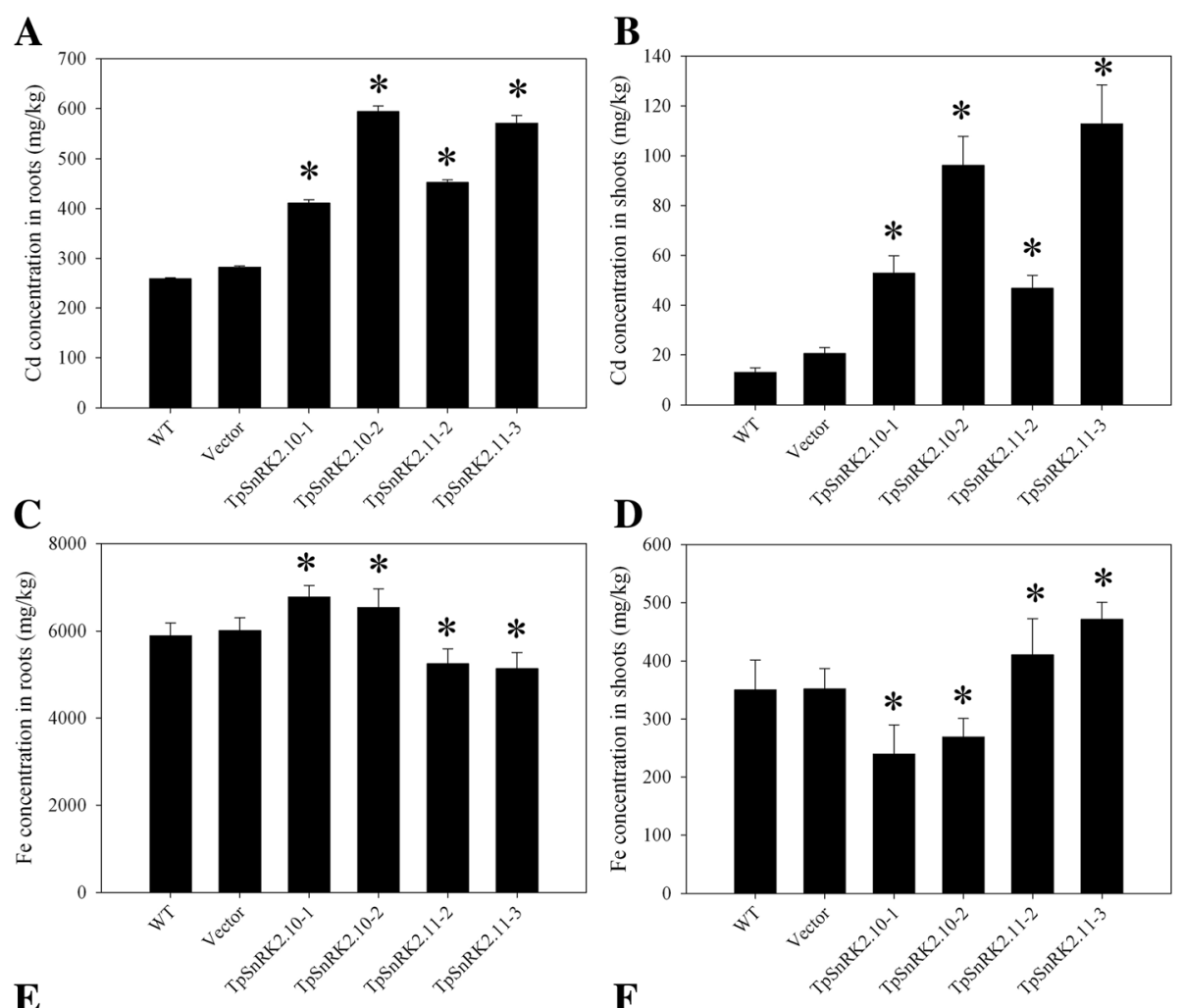

D
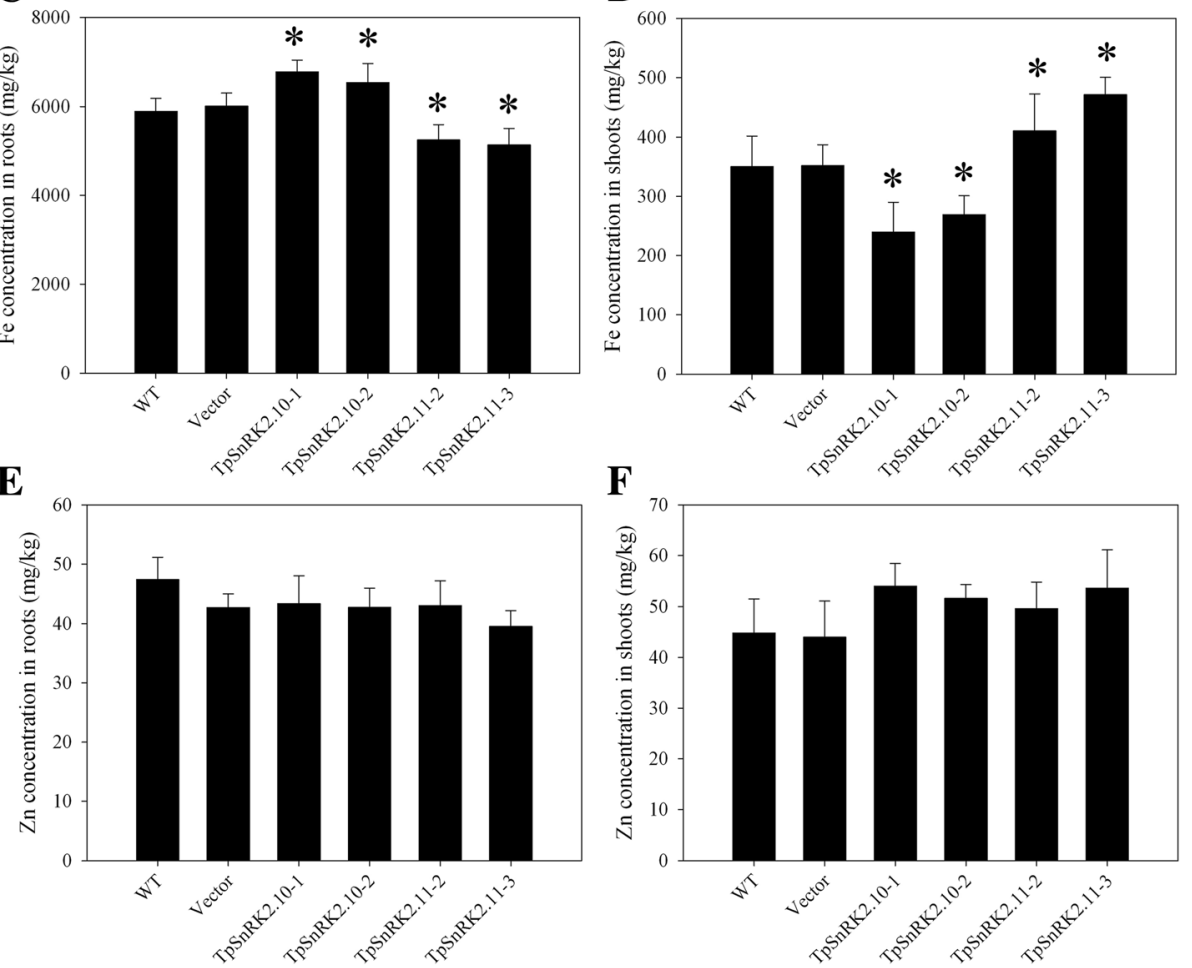

Fig. 6 Metal concentrations in roots and shoots of TpSnRK2.10- and TpSnRK2.11-overexpressing Arabidopsis. a-b Cd concentration in roots (a) and shoots (b); $\mathbf{c}-\mathbf{d}$ Fe concentration in roots (c) and shoots (d); e-f Zn concentration in roots (e) and shoots (f). Asterisk indicated significant difference when compared with WT at $P<0.05$; values were means \pm standard deviation (three biological replicates)

compared with WT and vector line (Fig. 6d); in contrast, overexpression of TpSnRK2.11 significantly reduced Fe concentration in roots (Fig. 6c), but enhanced in shoots when compared with WT and vector line (Fig. 6d). However, overexpression of TpSnRK2.10 or TpSnRK2.11 did not change $\mathrm{Zn}$ concentration in roots and shoots (Fig. 6e and f). Compared with WT, overexpression of TpSnRK2.10 or TpSnRK2.11 significantly enhanced Cd translocation factor (TF, the shoot-to-root $\mathrm{Cd}$ concentration ratio) (Fig. 7a); expression of TpSnRK2.11 enhanced Fe TF, while overexpression of $T p S n R K 2.10$ reduced that (Fig. $7 \mathrm{~b})$. These results indicated that TpSnRK2.10 and TpSnRK2.11 mediate Cd and Fe uptake and translocation.
To understand the physiological mechanisms that TpSnRK2.10 and TpSnRK2.11 participate in $\mathrm{Cd}$ and $\mathrm{Fe}$ uptake and translocation, we analyzed $\mathrm{Cd}$ and $\mathrm{Fe}$ subcellular distribution in overexpressing lines, WT and vector line. Compared with WT and vector line, overexpression of TpSnRK2.10 and TpSnRK2.11 dramatically increased $\mathrm{Cd}$ concentration of cell wall fraction and organelle fraction in roots and shoots (Fig. 8a and b). Overexpression of TpSnRK2.10 increased Fe concentration of cell wall fraction and organelle fraction in roots (Fig. 8c), but decreased that of cell wall fraction in shoots when compared with WT and vector line (Fig. 8d). However, overexpression of $T p S n R K 2.11$ reduced $\mathrm{Fe}$ 

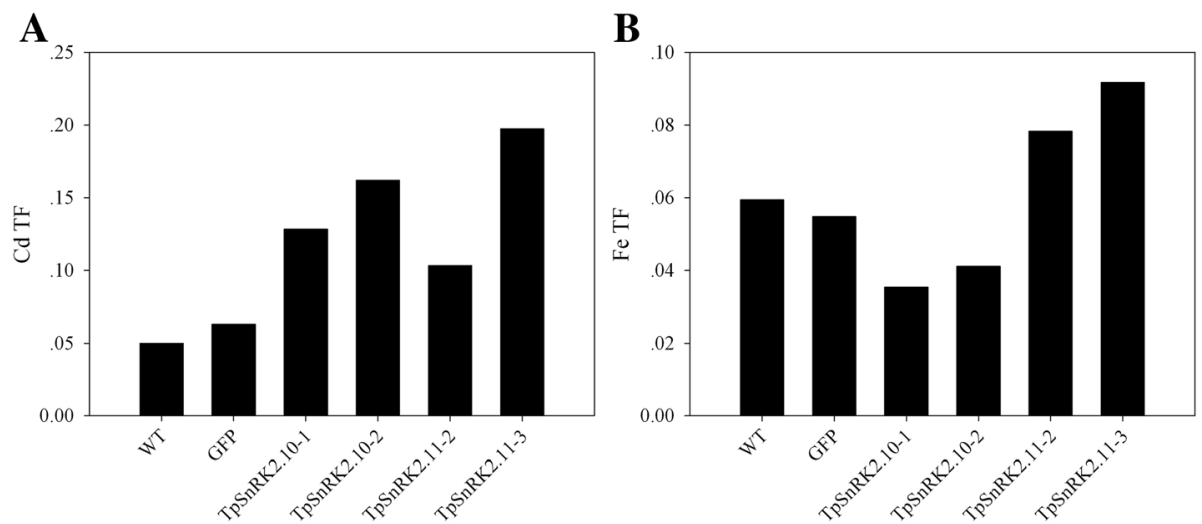

Fig. $7 \mathrm{Cd}$ and Fe translocation factors (TFs, the shoot-to-root Cd or Fe concentration ratio) of TpSnRK2.10- and TpSnRK2.11-overexpressing Arabidopsis. a Cd TF; B: Fe TF

concentration in soluble fraction in roots (Fig. 8c), and cell wall fraction in shoots (Fig. 8d). These results implied that TpSnRK2.10 and TpSnRK2.11 probably influence $\mathrm{Cd}$ and Fe uptake and translocation via changing $\mathrm{Cd}$ and Fe subcellular distribution in roots and shoots.
Additionally, we further analyzed the expression of AtIRT1, AtNRAMP1 and AtHMA4 in overexpressing lines to understand whether TpSnRK2.10 and TpSnRK2.11 change their expression. Interestingly, we found that overexpression of TpSnRK2.10 and TpSnRK2.11 did not influence the expression of AtIRT1 (Fig. 9a), but significantly
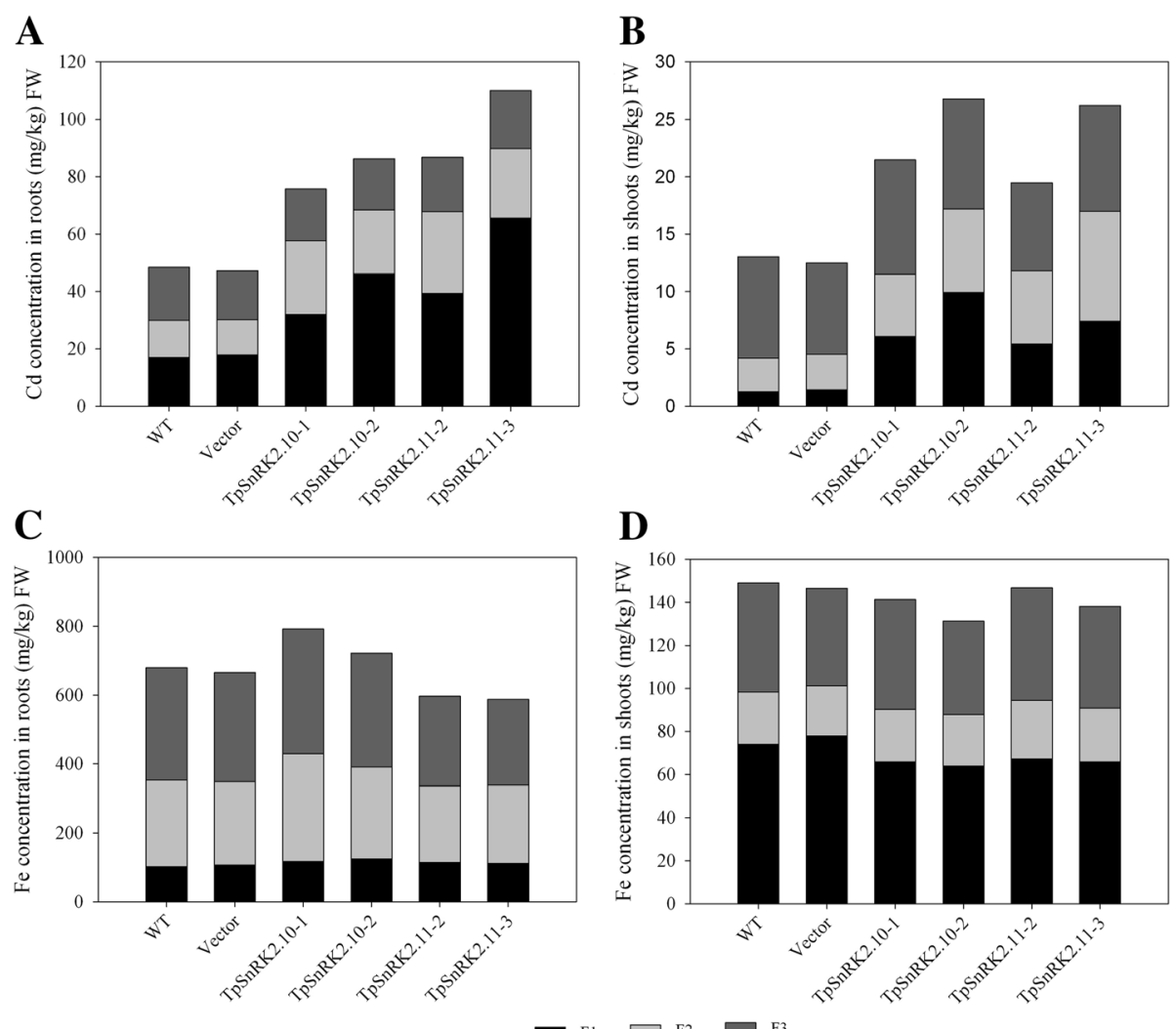

\section{D}

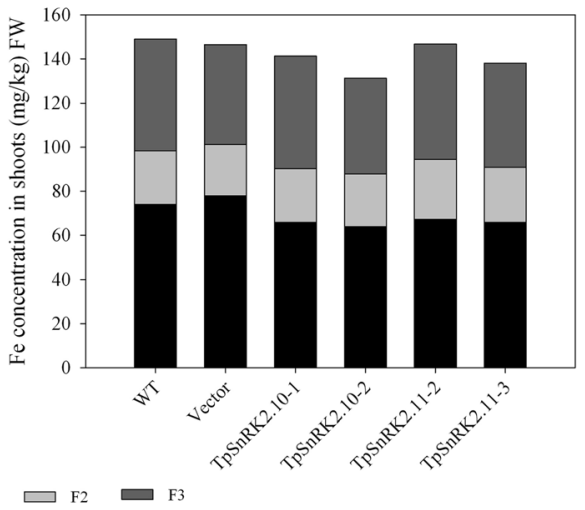

Fig. 8 Subcellular distribution of $\mathrm{Cd}$ and Fe in roots and shoots of TpSnRK2.10- and TpSnRK2.11-overexpressing Arabidopsis. a-b Cd concentration in roots (A) and shoots (B); c-d Fe concentration in roots (C) and shoots (D). All values were means derived from three biological replicates. F1 represents cell wall fractions; F2 represents organelle fractions; F3 represents soluble fractions 


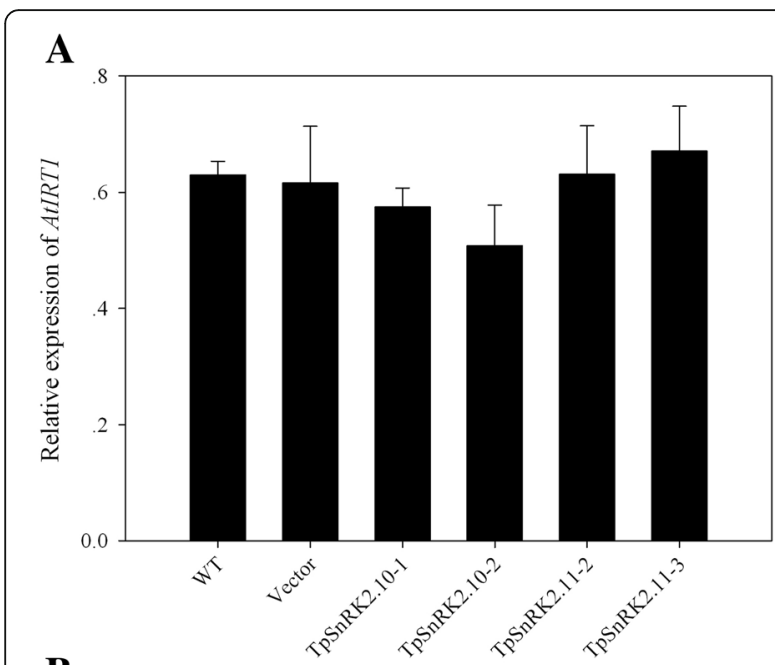

B

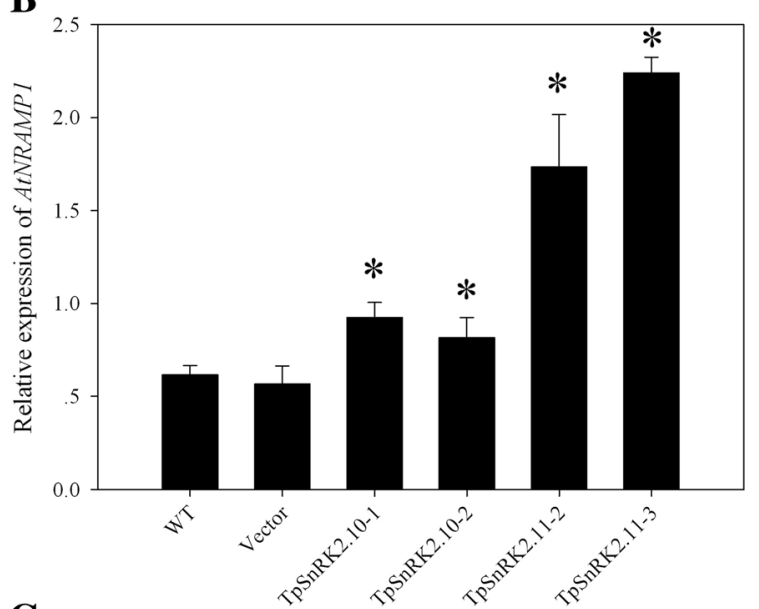

C

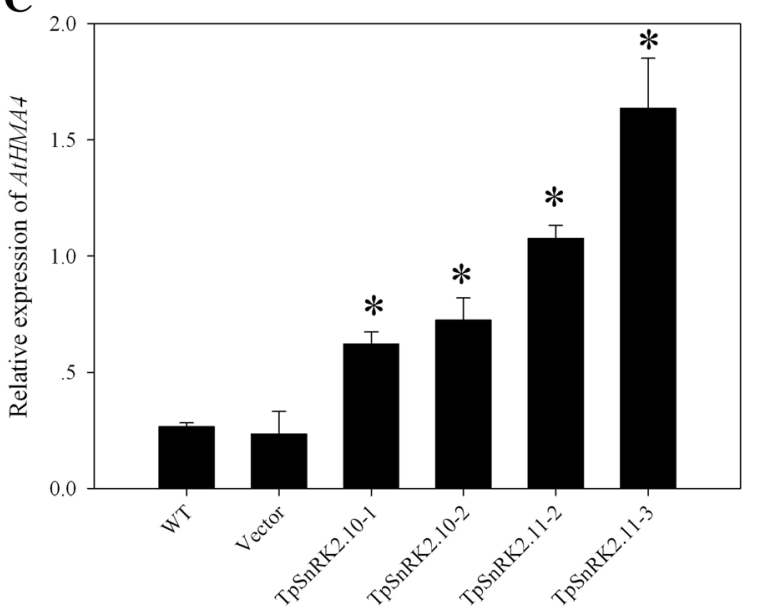

Fig. 9 Relative expression of AtIRT1, AtNRAMP1 and AtHMA4 in transgenic lines. a Relative expression of AtIRT1; $\mathbf{b}$ relative expression of AtNRAMP1; c relative expression of AtHMA4. Samples were collected from shoot of Arabidopsis without metal stress. Asterisk indicated significant different when compared with WT at $P<0.05$; value was mean \pm standard deviation (three biological replicates) enhanced the expression of AtNRAMP1 (Fig. 9b) and AtHMA4 (Fig. 9c).

\section{Discussion}

In Arabidopsis, Cd can activate AtSnRK2.4 and AtSnRK2.10; meanwhile, knockout of $A t S n R K 2.4$ enhances Cd tolerance [6]. Overexpression of wheat genes TaSnRK2.3, TaSnRK2.4, and TaSnRK2.7 or TaSnRK2.8 in Arabidopsis noticeably enhances drought, salinity and cold tolerance [18, 35-37]. Meanwhile, $\operatorname{SnRK2} 2$ from tetraploid wheat are differently regulated by several abiotic stresses including drought, salt, ABA and cold $[23,24]$. However, the roles of these SnRK2s from wheat in heavy metal responses and transport are still elusive. In this study, expression of TpSnRK2.11 restored the growth of $\mathrm{Cd}$ sensitive strain; while expression of $T p S n R K 2.10$ increased the growth inhibition of $\mathrm{Cd}$. The results suggested that TpSnRK2.10 and TpSnRK2.11 play different roles in $\mathrm{Cd}$ tolerance in yeast. Overexpression of $T p S n R K 2.10$ or TpSnRK2.11, however, did not change Cd tolerance in Arabidopsis, which was concluded from similar root growth and length of seedling under $40 \mu \mathrm{M} \mathrm{CdCl}_{2}$, and root and shoot dried weight of maturation under $40 \mathrm{mg} / \mathrm{kg}$ $\mathrm{CdCl}_{2}$. The discrepant results might result from the different genome buffering between yeast and Arabidopsis. Meanwhile, these results implied that the functions of TpSnRK2.10 and TpSnRK2.11 differ from that of AtSnRK2.4, which mediates Cd tolerance [6]. These differences might result from: (1) the low identity (68.6\%) between TpSnRK2.11 and AtSnRK2.4, which includes different residues in several crucial motifs (ATP binding site, activation loop, and serine/threonine protein kinases activity site); (2) the different subcellular localizations that TpSnRK2.11 was localized to the cytoplasm, but AtSnRK2.4 was localized to the cytoplasm and nucleus [6]; and (3) the different responses to $\mathrm{Cd}$, such as the expression of $T p S n R K 2.11$ was down-regulated by $\mathrm{Cd}$, and the expression of AtSnRK2.4 was up-regulated by $\mathrm{Cd}[6]$.

Many studies indicate that SnRK2 mediates the regulation of sucrose metabolism, phytochelatins synthesis, and sulfur metabolism [6, 11, 38], which play crucial roles in heavy metal binding, transport and tolerance $[16,19,39]$. Meanwhile, SnRK2s also regulate the expression of some genes that encode metal transporters and metal chelation, such as IRT and plant metallothionein $[6,12,40]$. Thus, SnRK2s would be involved in heavy metal uptake, translocation and tolerance. In Arabidopsis, knockout of AtSnRK2.2 and AtSnRK2.3 reduces $\mathrm{Cd}$ concentration in roots but increases that in shoots [12]; however, knockout of AtSnRK2.4 did not affect $\mathrm{Cd}$ concentration, although it enhanced $\mathrm{Cd}$ tolerance [6]. In this study, overexpression of TpSnRK2.10 or $T p S n R K 2.11$ significantly increased $\mathrm{Cd}$ concentration in roots and shoots, and enhanced $\mathrm{Cd}$ translocation. These results indicated that TpSnRK2.10 and TpSnRK2.11 are 
involved in Cd uptake and translocation in overexpressing Arabidopsis, which are different from the functions of AtSnRK2.2 and AtSnRK2.3 [12], and AtSnRK2.4 [6]. In Arabidopsis, AtIRT1 and AtNRAMP1 are major transporters for Cd uptake [13, 41]; their expression could be potentially mediated by SnRK2s through phosphorylation of ABFs [12]. In this study, overexpression of TpSnRK2.10 or TpSnRK2.11 in Arabidopsis enhanced the expression of AtNRAMP1 to increase $\mathrm{Cd}$ uptake. Meanwhile, overexpression of TpSnRK2.10 or TpSnRK2.11 in Arabidopsis also enhanced the expression of AtHMA4 that is responsible for $\mathrm{Cd}$ translocation in Arabidopsis [42], and enhanced Cd translocation from root-to-shoot.

Except transportation of Cd, AtNRAMP1 also transport $\mathrm{Fe}$ for its uptake [41]. Thus, overexpression of TpSnRK2.10 or TpSnRK2.11 in Arabidopsis enhanced Fe uptake and translocation. However, overexpression of $T p S n R K 2.10$ enhanced Fe concentration in roots, but reduced that in shoots, which implied that root retained more Fe to reduce Fe translocation. Conversely, overexpression of $T p S n R K 2.11$ reduced Fe concentration in roots, but increased that in shoots, which indicated that more Fe was transported into shoots. Thus, overexpression of TpSnRK2.10 and TpSnRK2.11 would differentially regulate other metal transporters that specifically transport Fe to mediate Fe translocation. For example, OsYSL15 only transports Fe for Fe uptake and translocation in rice [43]; HvYS1 specifically transports Fe for Fe uptake in barely [44]; and AtNRAMP3 pumps Fe from the vacuoles to the cytosol [45]. The different influences in $\mathrm{Fe}$ uptake and/or translocation implied that TpSnRK2.10 and TpSnRK2.11 have different biological functions, such as their different expression patterns.

Knockout of AtSnRK2.4 reduced the synthesis of PCs [6], and overexpression of AtSnRK2.6 increased the content of hemicellolosic polysaccharides of cell wall [11]. In plant, PCs chelate metals and then sequestrated into the vacuoles [16]; hemicellolosic polysaccharides chelate metals and binds in cell wall [19]. They affect metal binding and mobilization. In this study, overexpression of TpSnRK2.10 and TpSnRK2.11 might enhance the content of PCs and hemicellolosic polysaccharides. Thus, higher $\mathrm{Cd}$ concentration in cell wall fraction of roots and shoots was observed in overexpressing lines. The results implied that overexpression of TpSnRK2.10 and TpSnRK2.11 enhanced the require capacity of $\mathrm{Cd}$ in roots and shoots, resulting in the increased $\mathrm{Cd}$ concentration did not influence the growth. Meanwhile, increased $\mathrm{Fe}$ concentrations in cell wall and organelle fractions of roots were observed in TpSnRK2.10-overexpressing lines. Conversely, reduced Fe concentrations in soluble fraction of roots and cell wall fraction of shoots were detected in TpSnRK2.11-overexpressing lines. The changes of $\mathrm{Fe}$ concentration in roots and shoots were consistent with Fe translocation from roots to shoots. Thus, these results confirmed that TpSnRK2.10 and TpSnRK2.11 are involved in Cd and Fe distribution.

\section{Conclusion}

In summary, our results indicate that TpSnRK2.10 and TpSnRK2.11 are involved in $\mathrm{Cd}$ and $\mathrm{Fe}$ accumulation and distribution in overexpressing lines, possibly by regulating AtNRAMP1 and AtHMA4 who have ABF or $\mathrm{ABFs}$ element in their promoters, and/or regulating the synthesis of phytochelatins and hemicellolosic polysaccharides. Since overexpression of TpSnRK2.10 or $T p S n R K 2.11$ enhances Cd uptake and translocation from roots to shoots, knockout or knockdown of these two genes could help reduce $\mathrm{Cd}$ entrance into plant; or overexpression of these two genes could be used to phytoextract $\mathrm{Cd}$ from polluted soils.

\section{Additional file}

Additional file 1: Figure S1. Relative expression of TpSnRK2.10 (A) and TpSnRK2.11 (B) in transgenic Arabidopsis. (DOCX 89 kb)

\begin{abstract}
Abbreviations
ABA: abscisic acid; ABFs: ABRE binding factors; ABRE: ABA response element; Cd: cadmium; CK: control; DPW: dwarf Polish wheat; Fe: ferrum; GFP: green fluorescent protein; IRT1: IRON-REGULATED TRANSPORTER1; SD: synthetic defined; SnRK2: sucrose nonfermenting-1 (SNF1)-related protein kinase 2; WT: wild type
\end{abstract}

\section{Acknowledgements}

We thank the professor Yan Huang (College of Life Science, Sichuan Agricultural University) who provided the seed of Arabidopsis thaliana for transformation.

\section{Funding}

This work was supported by the National Natural Science Foundation of China (Grant No.: 31671688, 31670387 and 31870309). The funding body did not exert influence on the design of the study and collection, analysis, and interpretation of data and in writing the manuscript.

\section{Availability of data and materials}

The full length cDNA of TpSnRK2.10 (https://www.ncbi.nlm.nih.gov/nuccore/ KF688099.1/) and TpSnRK2.11 (https://www.ncbi.nlm.nih.gov/nuccore/ KF688100.1/) are available in NCBI GenBank with accessions KF688099 and KF688100, respectively. The amino sequences of AtSnRK2.2 (https://www. ncbi.nlm.nih.gov/protein/NP_001190047.1? report = genbank\&log $\$=$ protalign\&blast_rank $=1 \& R I D=7$ C1FFSHT014), AtSnRK2.3 (https://www.ncbi. $\mathrm{n} / \mathrm{m}$.nih.gov/protein/NP_201489.1? report=genbank\&log $\$=$ protalign\&blast_ rank=1\&RID=7C1J5MDD014) and AtSnRK2.4 (https://www.ncbi.nlm.nih.gov/ protein/NP_172563.1?report=genbank\&log $\$=$ protalign\&blast_rank=1\&RID= 7BZ7RNGH014) are obtained from NCBI GenPept with accessions NP_001190047, NP_201489 and NP_172563, respectively.

\section{Authors' contributions}

RW, CW, JZ and YW designed the work. RW, CW and QY did the Cd sensitive analysis, functional analysis in overexpressing Arabidopsis. XX and XF did the expression analysis. LS and HK did the transformation of Arabidopsis and the selection of homozygous overexpressing lines. $\mathrm{HZ}$ performed the subcellular localization of TpSnRK2.11. RW, CW, JZ, YZ and YW analyzed data and prepared Figs. RW, CW and $Y W$ wrote the manuscript. JZ, YZ and $Y W$ revised the manuscript. All authors read and approved the final manuscript. 


\section{Ethics approval and consent to participate}

The dwarf Polish wheat used here was collected from Tulufan region, Xinjiang province, China, by Professor Chi Yen (Triticeae Research Institute, Sichuan Agricultural University) in the 1980s. No permission was necessary to collect this sample. Professor Chi Yen undertook the formal identification of the sample. The voucher specimen and the seed are deposited in the Triticeae Research Institute, Sichuan Agricultural University, Chengdu, Sichuan, China. Collection of the dwarf Polish wheat complied with the institutional, national and international guidenlines.

\section{Consent for publication}

Not applicable.

\section{Competing interests}

The authors declare that they have no competing interests.

\section{Publisher's Note}

Springer Nature remains neutral with regard to jurisdictional claims in published maps and institutional affiliations.

\section{Author details}

${ }^{1}$ Triticeae Research Institute, Sichuan Agricultural University, Wenjiang 611130, Sichuan, China. ${ }^{2}$ Joint International Research Laboratory of Crop Resources and Genetic Improvement, Sichuan Agricultural University, Wenjiang 611130, Sichuan, China. ${ }^{3}$ College of Resources, Sichuan Agricultural University, Wenjiang 611130, Sichuan, China.

Received: 22 November 2018 Accepted: 6 March 2019

\section{Published online: 12 March 2019}

\section{References}

1. Perrier F, Yan B, Candaudap F, Pokrovsky OS, Gourdain E, Meleard B, et al. Variability in grain cadmium concentration among durum wheat cultivars: impact of aboveground biomass partitioning. Plant Soil. 2016;404(1-2):30720.

2. DalCorso G, Farinati S, Furini A. Regulatory networks of cadmium stress in plants. Plant Signal Behav. 2010;5(6):663-7.

3. Yang Y, Xiong J, Chen R, Fu G, Chen T, Tao L. Excessive nitrate enhances cadmium (cd) uptake by up-regulating the expression of OsIRT1 in rice (Oryza sativa). Environ Exp Bot. 2016;122(2016):141-9.

4. Wang $Y$, Wang $C$, Liu Y, Yu K, Zhou Y. GmHMA3 sequesters $c d$ to the root endoplasmic reticulum to limit translocation to the stems in soybean. Plant Sci. 2018;270(2018):23-9.

5. Farinati S, DalCorso G, Varotto S, Furini A. The Brassica juncea BjCdR15, an ortholog of Arabidopsis TGA3, is a regulator of cadmium uptake, transport and accumulation in shoots and confers cadmium tolerance in transgenic plants. New Phytol. 2010;185(4):964-78.

6. Kulik A, Anielska-Mazur A, Bucholc M, Koen E, Szymańska K, Żmieńko A, et al. SNF1-related protein kinases type2 are involved in plant responses to cadmium stress. Plant Physiol. 2012;160(2):868-83.

7. Cheng Y, Wang C, Chai S, Shuai W, Sha L, Zhang H, et al. Ammonium N influences the uptakes, translocations, subcellular distributions and chemical forms of $\mathrm{cd}$ and $\mathrm{Zn}$ to mediate the $\mathrm{cd} / \mathrm{Zn}$ interactions in dwarf polish wheat (Triticum polonicum L.) seedlings. Chemosphere. 2018;193(2018): 1164-71.

8. Yeh CM, Chien PS, Huang HJ. Distinct signaling pathways for induction of MAP kinase activities by cadmium and copper in rice roots. J Exp Bot. 2007; 58(3):659-71.

9. Liu X, Kim KE, Kim K, Nguyen XC, Han HJ, Jung MS, et al. Cadmium activates Arabidopsis MPK3 and MPK6 via accumulation of reactive oxygen species. Phytochemistry. 2010;71(5-6):614-8.

10. Fujii $\mathrm{H}$, Verslues $P E$, Zhu JK. Identification of two protein kinases required for abscisic acid regulation of seed germination, root growth, and gene expression in Arabidopsis. Plant Cell. 2007;19(2):485-94.

11. Zheng Z, Xu X, Crosley RA, Greenwalt SA. SunY, Blakeslee B, et al. the protein kinase SnRK2.6 mediates the regulation of sucrose metabolism and plant growth in Arabidopsis. Plant Physiol. 2010;153(1):99-113.

12. Fan SK, Fang XZ, Guan MY, Ye YQ, Lin XY, Du ST, et al. Exogenous abscisic acid application decreases cadmium accumulation in Arabidopsis plants, which is associated with the inhibition of IRT1-mediated cadmium uptake. Front Plant Sci. 2014;5:721.
13. Vert G, Grotz N, Dédaldéchamp F, Gaymard F, Guerinot ML, Briat JF, et al. IRT1, an Arabidopsis transporter essential for iron uptake from the soil and plant growth. Plant Cell. 2002;14(6):1223-33.

14. Thomine S, Wang R, Ward JM, Crawford NM, Schroeder Jl. Cadmium and iron transport by members of a plant metal transporter family in Arabidopsis with homology to Nramp genes. P Natl Acad Sci USA. 2000;97(9):4991-6.

15. Cailliatte R, Lapeyre B, Briat JF, Mari S, Curie C. The NRAMP6 metal transporter contributes to cadmium toxicity. Biochem J. 2009;422(2):217-28.

16. Brunetti P, Zanella L, De Paolis A, Di Litta D, Cecchetti V, Falasca G, et al. Cadmium-inducible expression of the ABC-type transporter AtABCC3 increases phytochelatin-mediated cadmium tolerance in Arabidopsis. J Exp Bot. 2015;66(13):3815-29.

17. Yoshida R, Umezawa T, Mizoguchi T, Takahashi S, Takahashi F, Shinozaki K. The regulatory domain of SRK2E/OST1/SnRK2.6 interacts with ABI1 and integrates abscisic acid (ABA) and osmotic stress signals controlling stomatal closure in Arabidopsis. J Biol Chem. 2006;281(8):5310-8.

18. Zhang H, Mao X, Wang C, Jing R. Overexpression of a common wheat gene TaSnRK2.8 enhances tolerance to drought, salt and low temperature in Arabidopsis. PloS One. 2010;5(12):e16041.

19. Song XQ, Liu LF, Jiang YJ, Zhang BC, Gao YP, Liu XL, et al. Disruption of secondary wall cellulose biosynthesis alters cadmium translocation and tolerance in rice plant. Mol Plant. 2013;6(3):768-80.

20. Johnson RR, Wagner RL, Verhey SD, Walke-Simmons MK. The abscisic acidresponsive kinase PKABA1 interacts with a seed-specific abscisic acid response element-binding factor, TaABF, and phosphorylates TaABF peptide sequences. Plant Physiol. 2002;130(2):837-46.

21. Kobayashi Y, Murata M, Minami H, Yamamoto S, Kagaya Y, Hobo T, et al. Abscisic acid-activated SNRK2 protein kinases function in the generegulation pathway of $A B A$ signal transduction by phosphorylating $A B A$ response element-binding factors. Plant J. 2005;44(6):939-49.

22. Zhang H, Li W, Mao X, Jing R, Jia H. Differential activation of the wheat SnRK2 family by abiotic stresses. Front Plant Sci. 2016;7:420.

23. Wang $Y$, Wang $X$, Gu M, Kang H, Zeng J, Fan $X$, et al. Cloning and characterization of four novel SnRK2 genes from Triticum polonicum. Biol Plantarum. 2015;59(2):211-9.

24. Jiang $Y$, Wang $Y$, Huang $Z$, Kang $H$, Sha $L$, Fan $X$, et al. Cloning and characterization of four TpSnRK2s from dwarf polish wheat. Biol Plantarum. 2017:61(4):601-10.

25. Wang $X$, Wang C, Sheng H, Wang Y, Zeng J, Kang H, et al. Transcriptomewide identification and expression analysis of $A B C$ transporters in dwarf polish wheat under metal stresses. Biol Plantarum. 2017;61(2):293-304.

26. The International Wheat Genome Sequencing Consortium. A chromosomebased draft sequence of the hexaploid bread wheat (Triticum aestivum) genome. Science. 2014;345(6194):1251788.

27. Peng F, Wang C, Zhu J, Zeng J, Kang H, Fan X, et al. Expression of TpNRAMP5, a metal transporter from polish wheat (Triticum polonicum L.), enhances the accumulation of $\mathrm{cd}, \mathrm{co}$ and $\mathrm{Mn}$ in transgenic Arabidopsis plants. Planta. 2018;247(6):1395-406.

28. Yoo SD, Cho YH, Sheen J. Arabidopsis mesophyll protoplasts: a versatile cell system for transient gene expression analysis. Nat Protoc. 2007;2(7): $1565-72$.

29. Clough SJ, Bent AF. Floral dip: a simplified method for Agrobacteriummediated transformation of Arabidopsis thaliana. Plant J. 1998;16(6):735-43.

30. Innatowicz A, Siwinska J, Meharg AA, Carey M, Koornneef M, Reymond M. Conserved histidine of metal transporter AtNRAMP1 is crucial for optimal plant growth under manganese deficiency at chilling. New Phytol. 2014; 202(4):1173-83.

31. Boonyaves K, Gruissem W, Bhullar NK. NOD promoter-controlled AtRT1 expression functions synergistically with NAS and FERRITIN genes to increase iron in rice grains. Plant Mol Biol. 2016;90(3):207-15.

32. Chen ZR, Kuang L, Gao YQ, Wang YL, Salt DE, Chao DY. AtHMA4 drives natural variation in leaf $\mathrm{Zn}$ concentration of Arabidopsis thaliana. Front Plant Sci. 2018;9:270.

33. Giniger $E$, Varnum SM, Ptashne M. Specific DNA binding of GAL4, a positive regulatory protein of yeast. Cell. 1985;40(4):767-74.

34. Wang Y, Yu KF, Poysa V, Shi C, Zhou YH. A single point mutation in GmHMA3 affects cadmium (cd) translocation and accumulation in soybean seeds. Mol Plant. 2012;5(5):1154-6.

35. Mao X, Zhang H, Tian S, Chang X, Jing R. TaSnRK2.4, an SNF1-type serine/ threonine protein kinase of wheat (Triticum aestivum L.), confers enhanced multistress tolerance in Arabidopsis. J Exp Bot. 2010;61 (3):683-96. 
36. Zhang H, Mao X, Jing R, Chang X, Xie H. Characterization of a common wheat (Triticum aestivum L.) TaSnRK2.7 gene involved in abiotic stress responses. J Exp Bot. 2011;62(3):975-88.

37. Tian S, Mao X, Zhang H, Cheng S, Zhai C, Yang S, et al. Cloning and characterization of TaSnRK2.3, a novel SnRK2 gene in common wheat. J Exp Bot. 2013;64(7):2063-80.

38. González-Ballester D, Pollock SV, Pootakham W, Grossman AR. The central role of a SNRK2 kinase in sulfur deprivation responses. Plant Physiol. 2008; 147(1):216-27.

39. Liang T, Ding H, Wang G, Kang J, Pang H, Lv J. Sulfur decreases cadmium translocation and enhances cadmium tolerance by promoting sulfur assimilation and glutathione metabolism in Brassica chinensis L. Ecotox Environ Safe. 2016;124(2016):129-37.

40. Nakashima K, Fujita Y, Kanamori N, Katagiri T, Umezawa T, Kidokoro S, et al. Three Arabidopsis SnRK2 protein kinases, SRK2D/SnRK2.2, SRK2E/SnRK2.6/ OST1 and SRK21.SnRK2.3, involved in ABA signaling are essential for the control of seed development and dormancy. Plant Cell Physiol. 2009;50(7): 1345-63.

41. Cailliatte R, Schikora A, Briat JF, Mari S, Curie C. High-affinity manganese uptake by the metal transporter NRAMP1 is essential for Arabidopsis growth in low manganese conditions. Plant Cell. 2010;22(3):904-17.

42. Wong CKE, Cobbett CS. HMA P-type ATPases are the major mechanism for root-to-shoot cd translocation in Arabidopsis thaliana. New Phytol. 2009; 181(1):71-8.

43. Lee S, Chiecko JC, Kim SA, Walker EL, Lee Y, Guerinot ML, et al. Disruption of OsYSL15 leads to iron inefficiency in rice plants. Plant Physiol. 2009;150(2): 786-800.

44. Murata Y, Ma JF, Yamaji N, Ueno D, Nomoto K, Iwashita T. A specific transporter for iron (III)-phytosiderophore in barely roots. Plant J. 2006;46(4): $563-72$.

45. Thomine S, Lelievre F, Debarbieux E, Schroeder Jl, Barbier-Brygoo H. AtNRAMP3, a multispecific vacuolar metal transporter involved in plant responses to iron deficiency. Plant J. 2003;34(5):685-92.

Ready to submit your research? Choose BMC and benefit from:

- fast, convenient online submission

- thorough peer review by experienced researchers in your field

- rapid publication on acceptance

- support for research data, including large and complex data types

- gold Open Access which fosters wider collaboration and increased citations

- maximum visibility for your research: over $100 \mathrm{M}$ website views per year

At $\mathrm{BMC}$, research is always in progress.

Learn more biomedcentral.com/submissions 